\title{
Response to real-life decision-making impact of oncotype DX
}

\author{
Federico Augustovski ${ }^{1,2} \cdot$ Natalie Soto ${ }^{1} \cdot$ \\ Joaquín Caporale $^{1} \cdot$ Lucas Gonzalez $^{1}$. \\ Luz Gibbons $^{1} \cdot$ Agustín Ciapponi $^{1}$
}

Published online: 16 October 2015

(c) Springer Science+Business Media New York 2015

To the Editor,

We were pleased to receive the feedback provided in the letter by Bertelli et al. about their additional clinical experience with the 21 gene essay -Oncotype DX. As mentioned by the authors, the larger effect they found in decision impact in the highly selected population they describe is in line with our own findings. Additionally, even though we did not find this same trend in net reduction of chemotherapy, heterogeneity between studies was high regarding this outcome, especially when analyzing the sparing chemotherapy effect in patients classified by the test as belonging to the intermediate risk group. The authors provide details on previous experience where other markers were found to be highly correlated with patients being classified as low or high risk, thus rendering the test not necessary and likely leading to a higher proportion of patients belonging to the intermediate group, where evidence regarding the effect of chemotherapy is less clear [1] and where local practice might be a factor of increased relevance. Further studies will probably refine these issues, and also help to better determine its cost-effectiveness, which has been favorably judged, as the author's state, by NICE in the UK.

\section{Reference}

1. Paik S, Tang G, Shak S et al (2006) Gene expression and benefit of chemotherapy in women with node-negative, estrogen receptorpositive breast cancer. J Clin Oncol 24(23):3726-3734

This rebuttal letter refers to the letter to the editor article available at doi: 10.1007/s10549-015-3600-3.

\footnotetext{
Federico Augustovski

faugustovski@iecs.org.ar

1 Instituto de Efectividad Clínica y Sanitaria, Buenos Aires, Argentina

2 Departamento de Salud Pública, Facultad de Medicina, Universidad de Buenos Aires, Buenos Aires, Argentina
} 